\title{
Hyperauthorship: A comparative study of genetics and high-energy physics research
}

\author{
Yu-Wei Changa ${ }^{1,2}$, Mu-Hsuan Huang ${ }^{1 *}$ and Min-Jy Chiu ${ }^{1}$ \\ ${ }^{1}$ Department of Library and Information Science, \\ National Taiwan University, Taipei, TAIWAN \\ ${ }^{2}$ Center for Research in Econometric Theory and Applications, \\ National Taiwan University, Taipei, TAIWAN \\ e-mail: yuweichang2013@ntu.edu.tw; \\ mhhuang@ntu.edu.tw* (corresponding author); \\ r01126002@ntu.edu.tw
}

\begin{abstract}
The number of hyperauthored articles has increased in certain disciplines; yet, little research has been conducted on hyperauthorship. Because genetics and high-energy physics are fields that exhibit hyperauthorship, this study focused on the differences and trends in the characteristics and influence of hyperauthored articles in these fields, for which articles published between 2004 and 2013 were used. The findings show that the percentage of hyperauthored articles in high-energy physics was higher than that of those in genetics. Although low small proportions of hyperauthored articles were identified in the two fields, increasing trends were observed in the annual percentages of hyperauthored articles. The average numbers of authors, institutions, and countries per hyperauthored article in high-energy physics were higher than those in genetics. Furthermore, increasing trends were identified in the annual average numbers of authors and institutions per hyperauthored article in both fields. The average numbers of citations received by hyperauthored articles were significantly greater than those of other coauthored articles in both fields. A higher percentage of hyperauthored articles in high-energy physics received more than 100 citations than did multiauthored articles. A similar phenomenon did not occur in genetics.
\end{abstract}

Keyword: Authorships; Hyperauthorship; Genetics; High-energy Physics; Collaborative research. 


\section{INTRODUCTION}

In the early 1960s, natural sciences research was conducted by research teams, which signals the existence of "big science" shifting from "little science" (Price 1986). Since then, numerous studies have revealed an increase in the number of authors per article across disciplines (Fernandes 2014; Fernández 1998; Gazni et al. 2012; Kapoor et al. 2015; Ojerholm and Swisher-McClure 2015). Multiauthorship, which refers to the coauthoring of papers, has become a research focus. The primary factors contributing to the prevalence of collaborative research include highly professionalized academic disciplines and research complexity (Beaver and Rosen 1978; Green and Johnson 2015). Disciplinary cultures also facilitate the formation of large research teams. For instance, research in physics, chemistry, and engineering has focused on the use of large government-funded laboratories (Hinnant et al. 2012). In addition, honorary authorship is a further possible explanation for the rapid increase in the number of authors per paper (Al-Herz et al. 2014; Dong et al. 2016; Kornhaber, McLean and Baber 2015; Ojerholm and Swisher-McClure 2015; Rajasekaran, Shan and Finnoff 2014; Slone 1996).

A substantial increase in research team size was observed not only in the natural sciences and technology but also in the social sciences (Wuchty, Jones and Uzzi 2007). In particular, research teams with 100 or more researchers have formed (Milojević 2014). The term "multiauthorship" is inadequate for describing the phenomenon of a vast number of authors of an article when the author numbers have exceeded most people's comprehension. In such a situation, the existence of hyperauthorship with more than 100 authors of an article and its problems in certain disciplines has been addressed (Cronin 2001; Greene 2007). The range of multiauthorship was narrowed to articles coauthored by 2-99 authors. In addition, the record for the number of authors of a single article continues to be broken. An article written by more than 1,000 authors was published in 2004 (King 2012). In 2015, a physics paper with 5,154 authors broke the record (Castelvecchi 2015). A notable spike in the number of hyperauthored articles appeared at the end of the 2000s (Hotz 2015). This implies that researchers can create new terms to refer to articles by, for example, 500 or 1000 authors. Hyperauthorship is the phenomenon in which a large number of authors contribute to a single article; it has existed for a substantial period and is not a new phenomenon.

Two subjects, genetics belonging to biological medicine and high-energy physics belonging to physics, are regarded as two representatives of hyperauthorship (King 2012; Patience et al. 2017). Although the number of physics articles coauthored by a huge number of authors is marginal, some of them were found to be written by more than 1,000 authors (Franceschet and Costantini 2010). Such a high number of authors per article explains why a higher 
average number of authors per article appears in physics compared with other fields. In biology, medicine, and physics, single-authored articles are rare (Franceschet and Costantini 2010). The emergence of a huge number of authors of an article in biological medicine and physics has been identified by numerous empirical studies (Birnholtz 2006; Constantian 1999; Cronin 2001; Franceschet and Costantini 2010; Greene 2007; Hall et al. 2008; Kreschmer and Rousseau 2001; Laudel 2002; Morris and Goldstein 2007; Patience et al. 2017).

Although the increasing trend in the number of hyperauthored articles has been observed, empirical studies related to hyperauthorship have been limited, focusing only on the numbers of hyperauthored articles and hyperauthorship disputes. The characteristics of hyperauthored articles have not been investigated. In addition, numerous studies have proved that coauthored articles have a greater influence than single-authored articles have, according to the number of citations received from other papers (Franceschet and Costantini 2010; Iribarren-Maestro, Lascurain-Sánchez, and Sanz-Casado 2009; Ma and Guan 2005), although the positive relationship between coauthorship and the influences of publications are not supported by all related studies (Avkiran 1997; Leimu and Koricheva 2005). These findings from previous related studies prompted us to investigate the influence of hyperauthored articles. Whether hyperauthored articles have greater influences compared with other coauthored articles was one of the focuses of this study. To understand the characteristics and influences of hyperauthored articles more clearly, this study focused on the trend of hyperauthorship in the two fields of genetics and high-energy physics. Three research questions were addressed in this study as follows:

(a) What trends exist in the annual proportion of hyperauthored articles in genetics and high-energy physics?

(b) What trends are observed in the average numbers of authors, institutions, and countries per hyperauthored article by year?

(c) What differences exist in the influence among hyperauthored articles, other coauthored articles, and single-authored articles?

\section{LITERATURE REVIEW}

Tracking the changes in a high number of authors per article, Regalado (1995) identified the increasing trends in four groups of articles by the number of authors per article, namely 51100, 101-200, 201-500, and at least 501 authors, on the basis of Science Citation Index (SCI) articles published between 1981 and 1993. The consistent findings that increasing numbers in four groups of articles by 51-100, 101-200, 201-500, and 501-999 were also confirmed by King (2012) on the basis of SCl articles published in 1998-2011. Furthermore, articles written by at least 1,000 authors formed the fifth observation groups in this study. This 
indicates that articles written by at least 1,000 authors were published during 1998-2001. Although articles by at least 1,000 authors accounted for the smallest proportion among the five groups of multiauthored articles, a substantial increase was identified in 2011 . The number of authors per article continued to grow.

Multiauthorship has long been used to refer to articles by two or more authors (Halperin, Scott, and George 1992), highlighting the differences between single-authored and coauthored articles (Abt 1984; Iribarren-Maestro, Lascurain-Sánchez and Sanz-Casado 2009; Mitchell 1961; Rousseau 1992). Some studies have further categorized multiauthored articles based on a specific range of author numbers. For example, Garg and Padhi (2002) and Ma and Guan (2005) have defined papers by three or four authors as multiauthored and those by five or more authors as megaauthored papers. Yukawa, Kitanaka, and Yokoyama (2014) referred to multiauthored papers as papers written by three to nine authors. However, the ever-increasing trend in a high number of authors per article has prompted the creation of terms. Cronin (2001) coined the term hyperauthorship to refer to articles by at least 100 authors, indicating that author inflation had grown exponentially by the end of $20^{\text {th }}$ century. Kretschmer and Rousseau (2001) used the term megaauthorship to describe articles coauthored by at least 100 authors. Morris and Goldstein (2007) used the term "mass recognition authorship" to refer to articles by several hundreds of authors. They also borrowed the concept of hyperauthorship from Cronin and defined it as articles written by 20 or more authors. They claimed that the definition of hyperauthorship is inconsistent across disciplines. This study adopted the definition of hyperauthorship proposed by Cronin, in which hyperauthored articles are those by at least 100 authors. Thus, multiauthorship was limited to articles by 2-99 authors.

Trends in authorship inflation have created concerns about authorship criteria. The requirements for authorship are not identical across disciplines. Authorship is formed by social customs (Cho and McKee 2002). To clarify the roles and responsibilities in becoming an author, two professional organizations in biological medicine and physics, the American Physics Association (APA) and the International Committee of Medical Journal Editors (ICMJE) have defined requirements of being authors (APA 2016; ICMJE 2016); however, the requirements are not noticed and obeyed by all journals and authors. Wager (2007) reported that 41 percent of 234 biological medicine journals had no quality requirements of authors, 14 percent had established their own guidelines for authorship, and only 9 percent required authors to state their contribution to their articles. In addition, not all authors agree on the guidelines for authorship of the ICMJE (Hoen, Walvoort and Overbeke 1998). No authoritative requirements of authorship are widely accepted and obeyed (Constantian 1999). 
The inconsistent requirements of authorship have resulted in some listed authors not contributing to the content of their articles and other authors contributing to the content of articles but not being listed as authors (Mowatt et al. 2002). Promiscuous coauthorship, such as honorary authors, gift authors, and ghost authors, is one factor explaining the everincreasing average number of authors per article, including the formation of hyperauthorship (Al-Herz et al. 2014; Bates et al. 2004; Rajasekaran et al. 2014). In many disciplines, the order of authorship reflects the difference in contribution. Obviously, hyperauthorship makes it difficult to identify who deserves credit for a publication (Hotz 2015).

Numerous studies have reported on the substantial proportions of honorary authors in medicine-related fields (Al-Herz et al. 2014; Flanagin et al. 1998; Kennedy, Barnsteiner, and Daly 2014; Mowatt et al. 2002; Rajasekaran et al. 2014; Wislar et al. 2011). Honorary authorship is regarded as a serious problem in the field of biomedicine (Davenport and Cronin 2001). Salager-Meyer et al. (2009) claimed that honorary authorship is the worst in the biomedicine field. Several possible reasons for honorary authorship have been discussed and can be divided into two main categories. First, authors are not aware of authorship guidelines (Kennedy et al. 2014), despite authorship guidelines having been established in several disciplines and differences existing among them (Osborne and Holland 2009). Second, some researchers have abused their power by adding honorary authors to author lists to help one another improve their research productivity (Osborne and Holland 2009; Pennock 1996). In some cases, well-known honorary authors have been added to author lists to increase the acceptance rate of manuscripts (Al-Herz et al. 2014).

Hyperauthorship is the characteristic of high-energy physics research because of disciplinary tradition. Authorship practices vary from discipline to discipline and even between subfields of the same discipline (Delfanti 2016; Patience et al. 2017). High-energy physics researchers conduct experiments by using advanced scientific instruments and through large-scale collaborations, consisting of hundreds or thousands of members from institutions worldwide. All members of a research collaboration are listed alphabetically as authors when papers are published by any member. This highlights equal contribution and a collective effort (Birnholtz 2008; Delfanti 2016). Therefore, in addition to promiscuous coauthorship, authorship tradition is a factor leading to growth in hyperauthorship.

\section{METHODOLOGY}

After a review of related literature, the two fields of genetics and high-energy physics were selected as the subjects for hyperauthorship analyses in this study. Genetics and high-energy physics have been reported to have high proportions and increasing trends of hyperautored 
articles (King 2012; Patience et al. 2017). The characteristics and influences of hyperauthored articles were the focuses of this study. Journal candidates were from journals listed in the two subject categories of "genetics and heredity" and "particles and fields physics" in the 2013 version of Journal Citation Reports. Because a high number of articles $(258,590)$ were published in journals of the two fields during the study period (2004-2013) and the standardization of author affiliations from hyperauthored articles was highly laborious, two journals were selected in each field for analyses after considering the sample size that was determined at a 95 percent confidence level and approximately 1 confidence interval. This ensured that although only two journals were analyzed for each field, the number of sample articles met the statistical requirement.

The four selected journals have to meet three requirements as follows. First, journals not listed annually in the two subject categories of "genetics and heredity" and "particles and fields physics" during 2004-2013 were excluded to ensure that the bibliographic records of English articles in a 10-year period in selected journals could be collected from the Web of Science. Second, according to the differences of journal productivity, journals had to publish at least 80 research articles per year during 2004-2013. Third, the remaining journals were in descending order according to the value of the impact factor, and the proportion of hyperauthored articles in 2013 was calculated for each of them. Journals including hyperauthored articles were identified. Not all journals with high impact factor tend to have higher proportions of hyperauthored articles. Therefore, two journals for each discipline were selected by considering the two criteria of a high impact factor and a high percentage of hyperauthored articles (Table 1). The four selected journals were a trade-off between high impact factors and high proportions of hyperauthored articles.

Table 1: Journal Titles Selected in this Study

\begin{tabular}{llcc}
\hline \hline \multirow{2}{*}{ Field } & Journal title & No. of & $\begin{array}{c}\text { Hyperauthored } \\
\text { articles }\end{array}$ \\
\hline \hline \multirow{2}{*}{ Genetics } & Nature Genetics & 2,035 & 9.04 \\
& American Journal of Human Genetics & 1,971 & 1.14 \\
\hline \hline High-energy & European Physical Journal C & 3,722 & 13.27 \\
physics & Astroparticle Physics & 965 & 9.18 \\
\hline \hline
\end{tabular}

Articles were divided into three groups according to the number of authors: single-authored articles, multiauthored articles by 2-99authors, and hyperauthored articles by at least 100 authors. In addition to the number of authors, the numbers of institutions and countries where authors' institutions were located were counted per article. The same institutions and 
countries were counted once per article. In addition, the institution names were standardized to improve the precision rate of calculating the number of institutions.

\section{RESULTS}

\section{Trends in Coauthorship}

Table 2 shows that coauthored articles were dominant in the two fields of genetics and highenergy physics. The annual proportions of coauthored articles related to high-energy physics ranged between 52.8 percent and 86.9 percent. An increasing trend was observed in the annual proportion of coauthored articles related to high-energy physics. In addition, a substantial increase was observed in the average number of authors per article. The average number of authors per article inflated to 80 authors in 2011 and peaked at 197.9 in 2012. Regarding genetics articles, single-authored articles were rare. Approximately 97.2 percent of articles were coauthored, which was much higher than that in high-energy physics (77.4\%). Except in 2013, the annual proportions of coauthored articles were between 98.3 percent and 100 percent. Although no notable changes were revealed in the annual proportions of coauthored articles, an upward trend in the average number of authors per article was observed.

Table 2: Changes in Coauthorship Characteristics of Articles in Two Fields by Year.

\begin{tabular}{|c|c|c|c|c|c|c|c|c|}
\hline Year & $\begin{array}{l}\text { No. of } \\
\text { articles }\end{array}$ & $\begin{array}{l}\text { No. of } \\
\text { coauthored } \\
\text { articles }\end{array}$ & $\begin{array}{l}\text { Percentage of } \\
\text { coauthored } \\
\text { articles }\end{array}$ & $\begin{array}{c}\text { Average number } \\
\text { of authors per } \\
\text { article }\end{array}$ & $\begin{array}{l}\text { No. of } \\
\text { articles }\end{array}$ & $\begin{array}{l}\text { No. of } \\
\text { coauthored } \\
\text { articles }\end{array}$ & $\begin{array}{l}\text { Percentage of } \\
\text { coauthored } \\
\text { articles }\end{array}$ & $\begin{array}{c}\text { Average number } \\
\text { of authors per } \\
\text { article }\end{array}$ \\
\hline 2004 & 746 & 394 & $52.8 \%$ & 23.2 & 415 & 410 & $98.8 \%$ & 11.5 \\
\hline 2005 & 429 & 322 & $75.1 \%$ & 24.2 & 377 & 373 & $98.9 \%$ & 11.5 \\
\hline 2006 & 355 & 295 & $83.1 \%$ & 35.2 & 415 & 408 & $98.3 \%$ & 11.4 \\
\hline 2007 & 463 & 349 & $75.4 \%$ & 28.1 & 418 & 418 & $100.0 \%$ & 14.8 \\
\hline 2008 & 401 & 332 & $82.8 \%$ & 19.7 & 416 & 413 & $99.3 \%$ & 16.4 \\
\hline 2009 & 432 & 318 & $73.6 \%$ & 24.6 & 358 & 357 & $99.7 \%$ & 23.9 \\
\hline 2010 & 404 & 349 & $86.4 \%$ & 80.0 & 349 & 346 & $99.1 \%$ & 27.8 \\
\hline 2011 & 420 & 372 & $88.6 \%$ & 137.5 & 324 & 324 & $100.0 \%$ & 27.6 \\
\hline 2012 & 517 & 446 & $86.3 \%$ & 197.6 & 447 & 446 & $99.8 \%$ & 28.5 \\
\hline 2013 & 512 & 445 & $86.9 \%$ & 152.1 & 495 & 405 & $81.8 \%$ & 35.5 \\
\hline Total & 4,679 & 3,622 & $77.4 \%$ & 73.1 & 4,014 & 3900 & $97.2 \%$ & 20.7 \\
\hline
\end{tabular}

\section{Trends in Hyperauthorship}

Table 3 shows that the proportions of hyperauthored articles in high-energy physics and 
genetics were limited. The proportion of hyperauthored articles in high-energy physics (8.9\%) was larger than that in genetics (2.3\%). The annual proportions of hyperauthored articles related to high-energy physics ranged between 5.5 percent and 12.8 percent, and those of hyperauthored articles related to genetics were between 0 percent and 5.2 percent. Although the proportions of hyperauthored articles in high-energy physics and genetics were limited, slightly increasing trends were identified in them.

Table 3: Changes in Percentages of Hyperauthored Articles by Year.

\begin{tabular}{ccccc}
\hline \hline & \multicolumn{2}{c}{ High-energy physics } & \multicolumn{2}{c}{ Genetics } \\
\cline { 2 - 5 } Year & $\begin{array}{c}\text { No. of } \\
\text { hyperauthored } \\
\text { articles }\end{array}$ & $\begin{array}{c}\text { Percentage of } \\
\text { hyperauthored } \\
\text { articles }\end{array}$ & $\begin{array}{c}\text { No. of } \\
\text { hyperauthored } \\
\text { articles }\end{array}$ & $\begin{array}{c}\text { Percentage of } \\
\text { hyperauthored } \\
\text { articles }\end{array}$ \\
\hline 2004 & 54 & $7.2 \%$ & 2 & $0.5 \%$ \\
2005 & 30 & $7.0 \%$ & 0 & $0.0 \%$ \\
2006 & 40 & $11.3 \%$ & 0 & $0.0 \%$ \\
2007 & 37 & $8.0 \%$ & 3 & $0.7 \%$ \\
2008 & 22 & $5.5 \%$ & 1 & $0.2 \%$ \\
2009 & 33 & $7.6 \%$ & 7 & $2.0 \%$ \\
2010 & 40 & $9.9 \%$ & 17 & $4.9 \%$ \\
2011 & 45 & $10.7 \%$ & 17 & $5.2 \%$ \\
2012 & 66 & $12.8 \%$ & 18 & $4.0 \%$ \\
2013 & 52 & $10.2 \%$ & 23 & $4.6 \%$ \\
\hline Total & 419 & $8.9 \%$ & 88 & $2.3 \%$ \\
\hline \hline
\end{tabular}

\section{Hyperauthorship by Level}

Table 4 shows the characteristics of hyperauthorship in the two fields from three levels of authorship: individuals, institutions, and countries. At the individual level, a hyperauthored article in high-energy physics was written by at least 101 authors and at most 3,171 authors. The range of the number of authors per high-energy physics article was much wider than that per genetics article. The average number of authors per hyperauthored article in 2013 was approximately 3.5 times of that in 2004. Institutional analyses yielded a similar finding. Authors were affiliated with at least four institutions and at most 3,943 institutions in a hyperauthored article. The range of the number of institutions in a hyperauthored article in high-energy physics was wider than that in genetics. The smallest discrepancy between highenergy physics and genetics could be anticipated from country analyses. Up to 2,204 authors from 43 countries coauthored a hyperauthored article related to high-energy physics. A hyperauthored article related to genetics was written by 437 authors representing 32 countries. 
Table 4: Numbers of Authors, Institutions, and Countries per Hyperauthored Article.

\begin{tabular}{lrr}
\hline \multicolumn{1}{c}{ Items } & High-energy physics & Genetics \\
\hline \hline No. of authors per hyperauthored article & $101-3171$ & $100-437$ \\
No. of institutions per hyperauthored article & $4-3943$ & $15-569$ \\
No. of countries per hyperauthored article & $1-43$ & $1-32$ \\
Average number of authors per hyperauthored article & 764.6 & 170.4 \\
Average number of institutions per hyperauthored article & 782.3 & 224.6 \\
Average number of countries per hyperauthored article & 19.6 & 15.3 \\
\hline \hline
\end{tabular}

Figure 1 shows the changes in the annual average numbers of authors, institutions, and countries based on hyperauthored articles in high-energy physics and genetics. A notable increase in the average number of authors per article related to high-energy physics was identified during 2010-2013. The changes in the annual average number of institutions per article were similar to those in the annual average number of authors per article. Although the annual average number of institutions per article was lower than the annual number of authors per article, a decreasing discrepancy between them was identified, meaning that authors tended to be affiliated with different institutions. Observing the trends in hyperauthored articles in genetics, slightly increasing trends were identified in both the annual average number of authors and institutions per article. However, the annual average number of institutions was higher than the annual average number of authors. This indicates that genetics authors were commonly affiliated with numerous institutions.

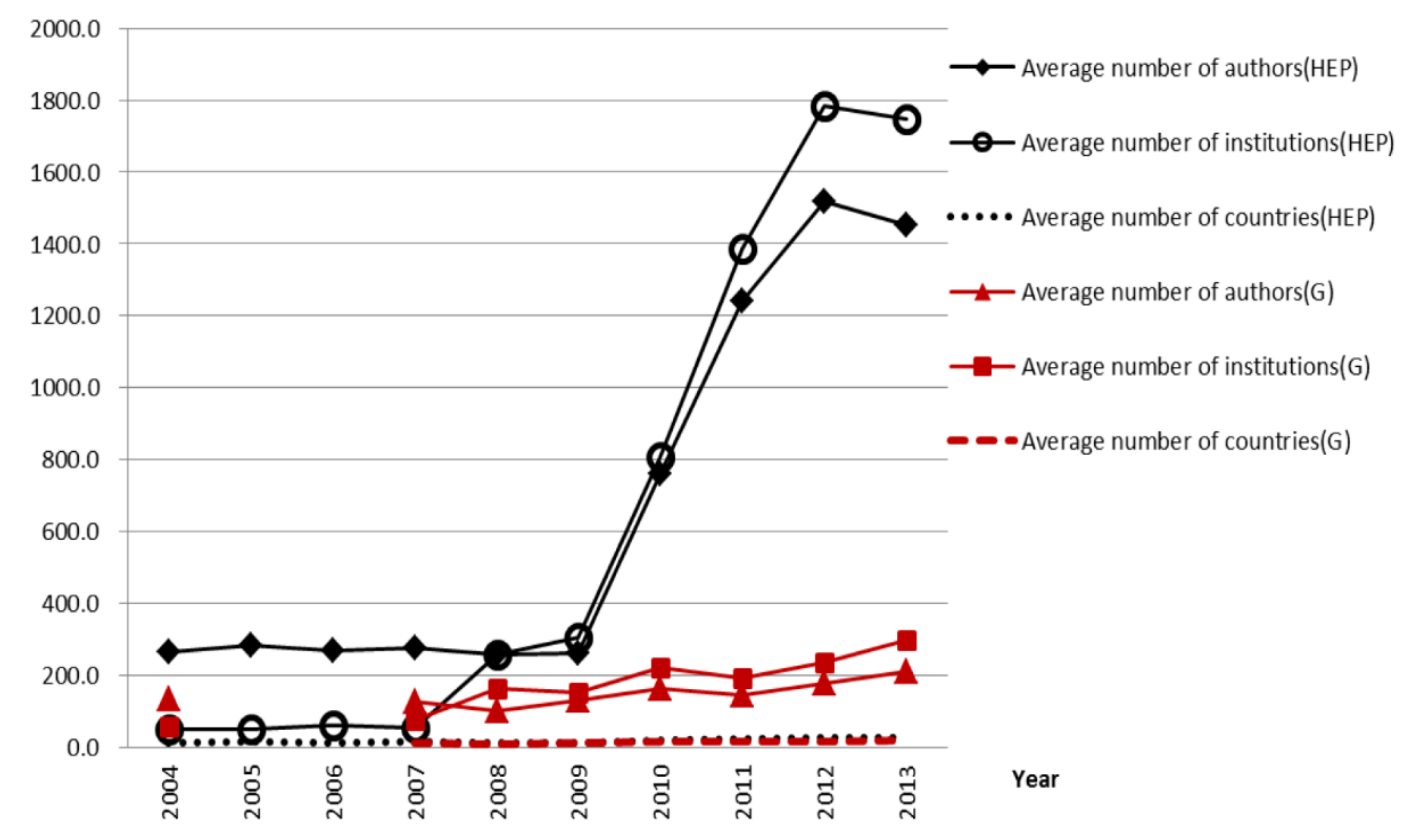

Figure 1: Changes in Average Number of Authors and Countries by Year and Discipline 
Regarding the average number of countries where authors' affiliations were located, no increasing or decreasing trends were observed in both genetics and high-energy physics. This reveals that authors of hyperauthored articles were concentrated within a certain range of countries. The authors of high-energy physics hyperauthored articles represented 73 countries and those of genetics hyperauthored articles represented 52 countries. Table 5 shows 12 countries with at least 50 percent of hyperauthored articles in both high-energy physics and genetics, demonstrating that authors of over half the hyperauthored articles represented 12 countries.

Table 5: Top countries involving at least half the hyperauthored articles.

\begin{tabular}{clrllrr}
\hline \hline \multicolumn{5}{c}{ High-energy physics } & & Genetics \\
\hline Rank & Country & No. of HA* & Percentage & Country & No. of HA* & Percentage \\
\hline \hline 1 & Italy & 390 & $90.3 \%$ & USA & 84 & $97.7 \%$ \\
2 & Germany & 386 & $89.4 \%$ & England & 83 & $96.5 \%$ \\
3 & England & 343 & $79.4 \%$ & Germany & 77 & $89.5 \%$ \\
4 & USA & 337 & $78.0 \%$ & Netherlands & 73 & $84.9 \%$ \\
5 & Russia & 324 & $75.0 \%$ & Sweden & 63 & $73.3 \%$ \\
6 & Switzerland & 321 & $74.3 \%$ & Finland & 62 & $72.1 \%$ \\
7 & France & 305 & $70.6 \%$ & Canada & 61 & $70.9 \%$ \\
8 & Poland & 303 & $70.1 \%$ & Italy & 57 & $66.3 \%$ \\
9 & Spain & 265 & $61.3 \%$ & Australia & 56 & $65.1 \%$ \\
10 & Greece & 247 & $57.2 \%$ & France & 55 & $64.0 \%$ \\
11 & Netherlands & 231 & $53.5 \%$ & Scotland & 52 & $60.5 \%$ \\
12 & Czech & 229 & $53.0 \%$ & Denmark & 43 & $50.0 \%$ \\
\hline \hline
\end{tabular}

*Hyperauthored articles

Approximately 90.3 percent of hyperauthored articles in high-energy physics were contributed by authors from Italy. Most Italian authors were affiliated with the Istituto Nazionale di Fisica Nucleare (National Institute for Nuclear Physics), a coordinating institution for nuclear, particle, and astroparticle physics in Italy. The National Institute for Nuclear Physics has sections in most major Italian universities and four national laboratories. In addition, up to 89.4 percent of hyperauthored articles in high-energy physics were published by authors from Germany. The Deutsches Elektronen-Synchrotron, the largest center for high-energy physics research in Germany, is the institution with highest number of authors of hyperauthored articles. Regarding authors of hyperauthored articles in genetics, at least 96.5 percent of articles were contributed by authors from the United States and England. Most American authors were affiliated with Harvard University, whereas most British authors were affiliated with the University of Oxford. 


\section{Influence of hyperauthored articles}

Among the three types of articles in genetics, the average number of citations received per hyperauthored article was highest (156.1). The same average number of citations per article appeared in multiauthored and single-authored articles (104.8). A one-way ANOVA test demonstrated that $F(2,3910)=4.16, p<.05$, showing that a significant difference existed among the three types of articles in terms of the average number of citations per article. Furthermore, Scheffe post hoc tests showed a significant difference between the pairs of means (156.1 vs. 104.8). Regarding articles in high-energy physics, the highest average number of citations was found in hyperauthored articles (21.0), followed by multiauthored articles (13.3) and single-authored articles (5.6). A significant difference was identified among the three groups of articles in high-energy physics $(F(2,4680)=84.65, p<.001)$. In addition, the Scheffe post hoc tests revealed significant differences in any pair of mean citations of the three groups of articles.

Figure 2 shows the annual average numbers of citations received by the three types of articles. Decreasing trends in the annual average numbers of citations per article were identified in the three types of articles related to high-energy physics. Except for those published in 2005 and 2006, hyperauthored articles obtained the highest annual average numbers of citations. Multiauthored articles received the second highest annual average number of citations except in 2004. Regarding genetics articles, three types of articles received a low number of citations each year. However, hyperauthored articles received the highest number of citations each year, followed by multiauthored articles and singleauthored articles. Slightly decreasing trends appeared in hyperauthored and multiauthored articles. No increasing or decreasing trend existed in single-authored articles.

Table 6 shows the distributions of citations received by the three groups of articles according to the number of authors per article in high-energy physics and genetics, namely hyperauthored, multiauthored, and single-authored articles. In high-energy physics articles, hyperauthored articles had the smallest proportion of articles with no citations (3.6\%) among the three types of articles. Most of the three types of articles received the same range of citations, between 1 and 100. Although a higher percentage of hyperauthored articles received more than 100 citations compared with the two other types of articles, only marginal discrepancies were observed among them. Regarding genetics articles, most of the three types of articles also received the same range of citations. However, a slightly higher percentage of multiauthored articles received more than 100 citations. 


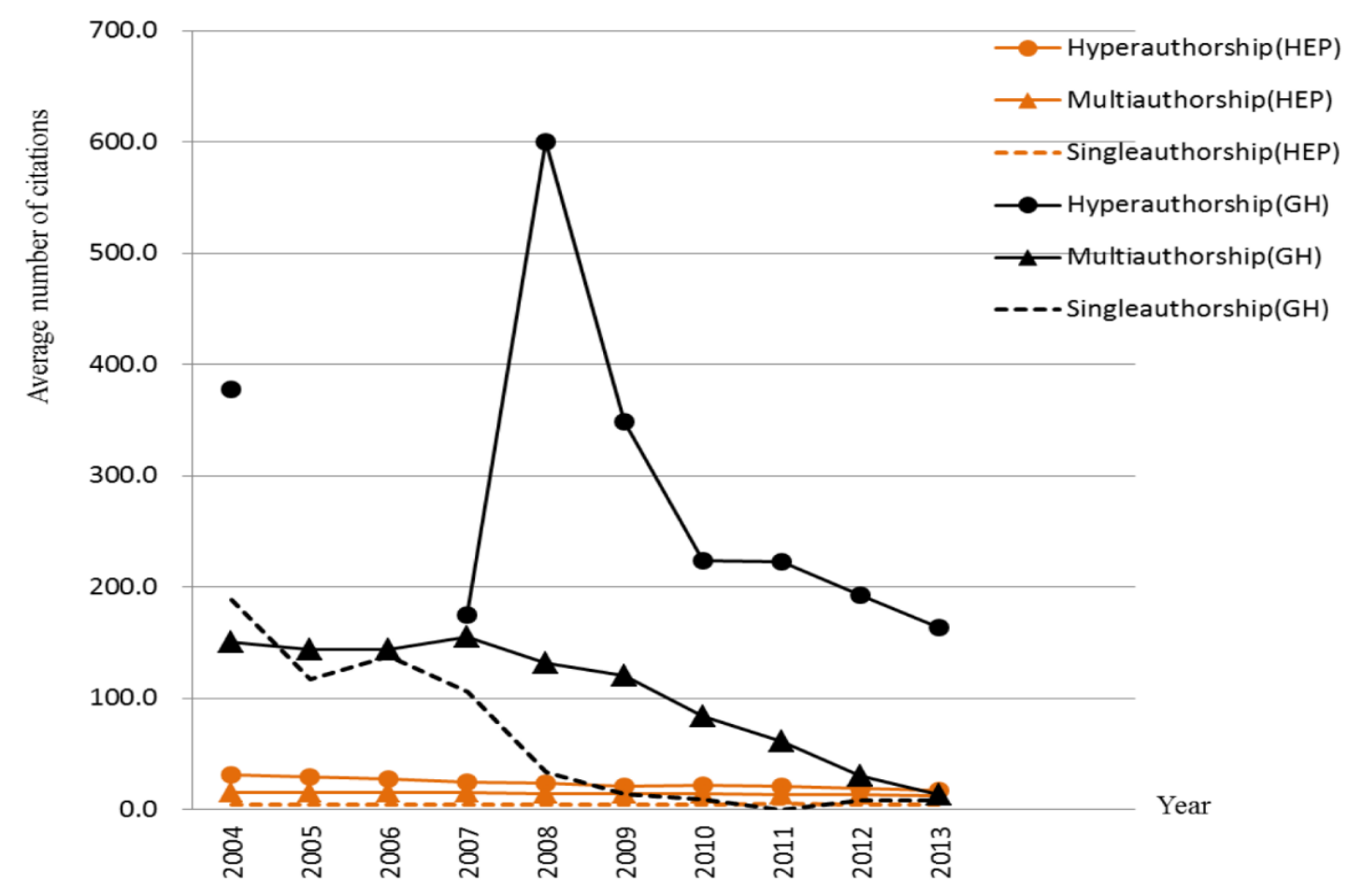

Figure 2: Average Number of Citations Received by Articles by Author Number

Table 6: Comparison of Citations Received by Three Types of Articles.

\begin{tabular}{|c|c|c|c|c|c|c|c|c|c|c|c|c|}
\hline \multirow{3}{*}{$\begin{array}{l}\text { No. of } \\
\text { citations }\end{array}$} & \multicolumn{6}{|c|}{ High-energy physics } & \multicolumn{6}{|c|}{ Genetics } \\
\hline & \multicolumn{2}{|c|}{$\begin{array}{c}\text { Hyper- } \\
\text { authored } \\
\text { articles }\end{array}$} & \multicolumn{2}{|c|}{$\begin{array}{l}\text { Multi- } \\
\text { authored } \\
\text { articles }\end{array}$} & \multicolumn{2}{|c|}{$\begin{array}{c}\text { Single- } \\
\text { authored } \\
\text { articles }\end{array}$} & \multicolumn{2}{|c|}{$\begin{array}{l}\text { Hyper- } \\
\text { authored } \\
\text { articles }\end{array}$} & \multicolumn{2}{|c|}{$\begin{array}{l}\text { Multi- } \\
\text { authored } \\
\text { articles }\end{array}$} & \multicolumn{2}{|c|}{$\begin{array}{c}\text { Single- } \\
\text { authored } \\
\text { articles }\end{array}$} \\
\hline & No. & $\%$ & No. & $\%$ & No. & $\%$ & No. & $\%$ & No. & $\%$ & No. & $\%$ \\
\hline 0 & 15 & 3.6 & 307 & 9.6 & 349 & 32.4 & -- & -- & 9 & 0.2 & -- & -- \\
\hline $1 \sim 100$ & 393 & 93.8 & 2849 & 89.4 & 728 & 67.5 & 52 & 58.4 & 2550 & 67.1 & 22 & 81.5 \\
\hline $101-200$ & 8 & 1.9 & 23 & 0.7 & 1 & 0.1 & 15 & 16.9 & 727 & 19.1 & 2 & 7.4 \\
\hline $201-300$ & 2 & 0.5 & 7 & 0.2 & -- & -- & 5 & 5.7 & 284 & 7.5 & 1 & 3.7 \\
\hline $301-400$ & 1 & 0.2 & -- & -- & -- & -- & 7 & 7.9 & 96 & 2.5 & -- & -- \\
\hline $401-500$ & -- & -- & 1 & -- & -- & -- & 2 & 2.2 & 44 & 1.2 & 1 & 3.7 \\
\hline $501-600$ & -- & -- & -- & -- & -- & -- & 2 & 2.2 & 37 & 1.0 & -- & -- \\
\hline $601-700$ & -- & -- & -- & -- & -- & -- & 2 & 2.2 & 12 & 0.3 & -- & -- \\
\hline $701-800$ & -- & -- & -- & -- & -- & -- & 4 & 4.5 & 17 & 0.4 & 1 & 3.7 \\
\hline $801-900$ & -- & -- & -- & -- & -- & -- & -- & -- & 5 & 0.1 & -- & -- \\
\hline $901-1000$ & -- & -- & -- & -- & -- & -- & -- & -- & 8 & 0.2 & -- & -- \\
\hline 1001- & -- & -- & -- & -- & -- & -- & -- & -- & 11 & 0.3 & -- & -- \\
\hline Total & 419 & 100.0 & 3187 & 100.0 & 1078 & 100.0 & 89 & 100.0 & 3800 & 100.0 & 27 & 100.0 \\
\hline
\end{tabular}




\section{DISCUSSION}

This study investigated the authorship characteristics of hyperauthored articles in the two fields of genetics and high-energy physics. The trend in hyperauthorship in both fields was one focus of this study. Although coauthored articles were the dominant type in the two fields, large discrepancies in the coauthorship rates were identified. In particular, it was rare for genetics researchers to publish single-authored articles. However, a higher coauthorship rate in genetics than in high-energy physics does not mean a higher percentage of hyperauthored articles or a higher average number of authors per hyperauthored article in genetics. Disciplinary cultures may explain the greater number of authors per article and the higher percentage of hyperauthored articles in high-energy than in genetics. Collective authorship with a long author list and equal contribution is traditional in high-energy physics (Birnholtz 2008; Delfanti 2016). Davenport and Cronin (2001) mentioned that high-energy physics research requires a large amount of capital and large-scale multidisciplinary collaboration. For disciplines focusing on facilities and instruments, most related research institutions cannot afford expensive instruments. Therefore, international collaboration is a common approach to providing research teams with facilities and instruments. The research institutions with the most-advanced facilities and instruments typically gather research teams from around the world to work on large international scientific projects. All collaborating members are listed as authors, and members are not allowed to submit their manuscripts for publication individually (Delfanti 2016).

Although a larger proportion of hyperauthored articles was revealed in high-energy physics compared with genetics ( $8.9 \%$ vs. $2.3 \%$ ), the hyperauthorship rates were marginal in both In addition, similar trends were observed in the two fields, including increasing trends in the annual percentage of hyperauthored articles and the annual average number of authors per hyperauthored article. This indicates that hyperauthored articles were published at an increasing rate and the size of research teams tended to grow. A trend of authors coming from a wide range of institutions in numerous countries can be anticipated. Gazni et al. (2012) investigated the size of research teams and reported that the size of teams showed large variation between fields. The largest teams have become more diverse than the smaller teams have and tend more toward interinstitutional and international collaboration.

Regarding the influence of hyperauthored articles, a slightly higher percentage of hyperauthored articles received at least 101 citations than did multiauthored articles in highenergy physics ( $2.6 \%$ vs. $0.9 \%$ ). A different situation was observed in genetics, in that a higher percentage of multiauthored articles received at least 101 citations than did hyperauthored articles (41.6\% vs. 33.7\%). The findings support those of Onodera and Yoshikane (2015), in that the number of authors was not a primary factor affecting the number of citations of 
articles. However, the citations received by articles in this study were collected on July 31 , 2014. Time being a primary factor affecting the accumulated number of citations received by articles explains why the average numbers of citations received by articles in 2012 and 2013 were lower than those in previous years.

Possible explanations for increasing hyperauthorship include increasingly complex and collaborative research, disciplinary tradition, and honorary authorship. Papatheodorou et al. (2008) claimed that the research topic was a strong determinant of the number of authors. Other possible factors facilitating scientific collaboration may include enhanced productivity, visibility, and communication (Bordons et al. 1996; Fernández 1998). Among numerous possible factors, the primary factors having a strong association with hyperauthorship require further investigation. However, the reliance on advanced instruments and large research collaborations explains the increase in the number of authors per hyperauthored article. In this study, the high-energy physics articles by at least 1000 authors are typically large interdisciplinary research collaborations, consisting of research teams from numerous institutions and countries. Furthermore, honorary authorship has been mentioned as a possible primary factor violating academic ethics. Although in numerous disciplines journals have an authorship policy (Resnik et al. 2016; Resnik and Master 2011), inconsistent authorship requirements have prevented researchers from understanding the meaning of coauthorship (da Silva and Dobránszki 2016; Tarnow, de Young, and Cohen 2004).

Inappropriate multiple authorship leads to dilution of authorship responsibility and unjustified citations (Drenth, 1996). To prevent inappropriate authorship, researchers have suggested that each author must report individual contributions (Drenth 1996; Feeser and Simon 2008; Ojerholm and Swisher-McClure 2015; Wislar et al. 2011) or limit the number of authors in an article (Baek et al. 2015). According to the author guides of the four selected journals in this study, Nature Genetics requires author contribution statements and American Journal of Human Genetics adopts the author policy made by ICMJE. By contrast, two selected high-energy physics journals have no authorship statements. Authorship practices in high-energy physics dictate that author contribution statements are unnecessary becasue the discipline prefers to focus on equal contributions. This may be one possible reason for why the two selected high-energy physics journals had a higher average number of authors per article than did the two selected genetics journals. However, surveys have demonstrated that only a few journals require authors to describe individual contributions (Wager 2007). The claims of authorship contributions cannot be verified by editors or publishers (da Silva and Dobránszki 2016). Despite a few journals having limited the maximum number of authors for an article, this cannot reduce authorship inflation (Baek et al. 2015; Dong et al. 2016). Numerous surveys on the prevalence of honorary authorship have demonstrated that 
no effective solutions to honorary authorship have been developed (Al-Herz et al. 2014; Bonekamp et al. 2012; Jawaid and Jawaid 2013; Kennedy et al. 2014; Vera-Badillo et al. 2016; Vinther and Rosenberg 2012; Wislar et al. 2011). Honorary authorship is an ethical problem and relies on researchers' awareness.

In addition, each author being given the same contribution and credit is common in the field of medicine (Jia et al. 2016). This indicates that the number of authors of a medicine-related article does not affect the credit received by an individual author. In particular, the primary factor leading to the prevalence of honorary authorship is that researchers aim to improve research productivity. This is because research productivity is associated with promotion, tenure, and grant funding (Al-Herz et al. 2014). Honorary authors are usually senior or wellknown researchers and are regarded as influential researchers who facilitate the acceptance of a manuscript. Irrespective of honorary authors not having made substantial contributions to a publication, some researchers accept the existence of honorary authors (Yukawa et al. 2014). In such a situation, hyperauthorship becomes a useful strategy for helping researchers improve academic performance. Although some institutions review only first- or singleauthor publications to evaluate an author's research performance for promotion (Pritychenko 2016), the impact of such an assessment on hyperauthorship remains uncertain.

The primary limitation of this study is that only four journals were analyzed. Therefore, the results cannot be generalizable to other high-energy physics or genetics journals. Hyperauthored articles were rarely found in most genetics and high-energy physics journals, and identifying hyperauthored articles and standardizing authors' affiliated institutions among a large number of articles is highly laborious. Thus, only two high-energy physics journals and two genetics journals with a larger proportion of hyperauthored articles were analyzed. However, the selected journals have a higher percentage of hyperauthored articles and higher impact factors, the findings of this study can reflect the characteristics of hyperauthorship in journals with higher visibility in genetics and high-energy physics.

\section{CONCLUSION}

Physics and biomedicine are two widely mentioned disciplines with a high number of hyperauthored articles; however, this study determined that high-energy physics has a more apparent nature of hyperauthorship than does genetics. In addition to the huge number of authors of an article, hyperauthored articles were not observed to have higher visibility compared with other articles. The low percentage of hyperauthored articles shows that hyperauthorship is still an atypical type of research collaborations. Although a low percentage of hyperauthored articles in the two fields of high-energy physics and genetics 
was identified, increasing trends were observed.

The ever-increasing number of authors of a hyperauthored article has made researchers focus on inappropriate authorship. Although disciplinary natures in genetics and high-energy physics play an essential role in forming hyperauthorship, inappropriate authorship was considered to facilitate the growth of hyperauthored articles as well. Therefore, hyperauthored articles must continue to be monitored. Although hyperauthored articles have been published, related literature shows that the percentage of hyperauthored articles is still low. In addition to the possible continuing increase in the number of hyperauthored articles, whether hyperauthorship extends to other disciplines, what factors elevate the growth of hyperauthored articles, and the researchers' perceptions of hyperauthorship are further research focuses. Recognizing the primary factors that contribute to the increase of hyperauthored articles can assist the identification of whether the prevalence of honorary authorship is associated with hyperauthorship.

\section{ACKNOWLEDGEMENT}

This work was financially supported by the Center for Research in Econometric Theory and Applications (Grant no. 107L900204) from The Featured Areas Research Center Program within the framework of the Higher Education Sprout Project by the Ministry of Education (MOE) in Taiwan, and by the Ministry of Science and Technology (MOST), Taiwan, under Grant No. MOST 107-3017-F-002-004-.

\section{REFERENCES}

Abt, H. 1984. Citations to single and multiauthored papers. Publications of the Astronomical Society of the Pacific, Vol.96, no.583: 746-749.

Al-Herz, W., Haider, H., Al-Bahhar, M. and Sadeq, A. 2014. Honorary authorship in biomedical journals: how common is it and why does it exist? Journal of Medical Ethics, Vol.40, no.5: 346.

APA. 2016. Ethics and values: 02.2 APS guidelines for professional conduct. Available at: https://www.aps.org/policy/statements/02_2.cfm.

Avkiran, N. 1997. Scientific collaboration in finance does not lead to better quality research. Scientometrics, Vol.39, no.2: 173-184. Available at: https://doi.org/10.1007/BF02457446.

Baek, S., Yoon, D.Y., Cho, Y.K., Yun, E.J., Seo, Y.L., Lim, K.J. and Choi, C.S. 2015. Trend toward an increase in authorship for leading radiology journals. American Journal of Roentgenology, Vol.205, no.5: 924-928. Available at: 
https://doi.org/10.2214/AJR.15.14979.

Bates, T., Anić, A., Marusić, M. and Marusić, A. 2004. Authorship criteria and disclosure of contributions: comparison of 3 general medical journals with different author contribution forms. JAMA, Vol.292, no.1: 86-88.

Beaver, D D. and Rosen, R. 1978. Studies in scientific collaboration part I.: The professional origins of scientific co-authorship. Scientometrics, Vol.1, no.1: 65-84. Available at: https://doi.org/10.1007/BF02016840.

Birnholtz, J. 2006. What does it mean to be an author? The intersection of credit, contribution, and collaboration in science. Journal of the American Society for Information Science and Technology, Vol.57, no.13: 1758-1770. Available at: https://doi.org/10.1002/asi.20380.

Birnholtz, J. 2008. When authorship isn't enough: Lessons from CERN on the implications of formal and informal credit attribution mechanisms in collaborative research. Journal of Electronic Publishing, Vol.11, no.1. Available at: http://dx.doi.org/10.3998/3336451.0011.105.

Bonekamp, S., Halappa, V.G., Corona-Villalobos, C.P., Mensa, M., Eng, J., Lewin, J.S. and Kamel, I.R. 2012. Prevalence of honorary coauthorship in the American Journal of Roentgenology. American Journal of Roentgenology, Vol.198, no.6: 1247-1255. Available at: https://doi.org/10.2214/AJR.11.8253.

Bordons, M., Gómez, I., Fernández, M., Zulueta, M. and Méndez, A. 1996. Local, Domestic and International Scientific Collaboration in Biomedical Research. Scientometrics, Vol.37, no.2: 279-295. Available at: https://doi.org/10.1007/BF02093625.

Castelvecchi D. 2015. Physics paper sets record with more than 5,000 authors. Available at: http://www.nature.com/news/physics-paper-sets-record-with-more-than-5-000authors-1.17567.

Cho, M. and McKee, M. 2002. Authorship in biomedical research: Realities and expectations. Available at: http://www.sciencemag.org/careers/2002/03/authorship-biomedicalresearch-realities-and-expectations.

Constantian, M.B. 1999. The Gordian knot of multiple authorship. Plastic and Reconstructive Surgery, Vol.103, no.7: 2064-2066. Available at: https://doi.org/10.1097/00006534199906000-00044.

Cronin, B. 2001. Hyperauthorship: A postmodern perversion or evidence of a structural shift in scholarly communication practices? Journal of the American Society for Information Science and Technology, Vol.52, no.7: 558-569. Available at: https://doi.org/10.1002/asi.1097.

da Silva, J.A.T. and Dobránszki, J. 2016. Multiple authorship in scientific manuscripts: ethical challenges, ghost and guest/gift authorship, and the cultural/disciplinary perspective. Science and Engineering Ethics, Vol.22, no.5: 1457-1472. Available at: 
https://doi.org/10.1007/s11948-015-9716-3.

Davenport, E. and Cronin, B. 2001. Who dunnit? Metatags and hyperauthorship. Journal of the American Society for Information Science and Technology, Vol.52, no.9: 770-773. Available at: https://doi.org/10.1002/asi.1123.

Delfanti, A. 2016. Beams of particles and papers: How digital preprint archives shape authorship and credit. Social Studies of Science, Vol.46, no.4: 629-645. Available at: https://doi.org/10.1177/0306312716659373.

Dong, Y., Wang, P., Guo, L. and Liu, H. 2016. "Listing author contribution" does not alter the author inflation in the publications in basic research in four major gastroenterology journals in 10 years. Scientometrics, Vol.107, no.3: 1501-1507. Available at: https://doi.org/10.1007/s11192-016-1923-4.

Drenth, J. 1996. Proliferation of authors on research reports in medicine. Science and Engineering Ethics, Vol.2, no.4: 469-480. Available at: https://doi.org/10.1007/BF02583933.

Feeser, V.R. and Simon, J.R. 2008. The ethical assignment of authorship in scientific publications: issues and guidelines. Academic Emergency Medicine, Vol.15, no.10: 963969. Available at: https://doi.org/10.1111/j.1553-2712.2008.00239.x.

Fernandes, J. 2014. Authorship trends in software engineering. Scientometrics, Vol.101, no.1: 257-271. Available at: https://doi.org/10.1007/s11192-014-1331-6.

Fernández, J. 1998. The transition from an individual science to a collective one: The case of astronomy. Scientometrics, Vol.42, no.1: 61-74. Available at: https://doi.org/10.1007/BF02465012.

Flanagin, A., Carey, L.A., Fontanarosa, P.B., Phillips, S.G., Pace, B.P., Lundberg, G.D. and Rennie, D. 1998. Prevalence of articles with honorary authors and ghost authors in peerreviewed medical journals. JAMA, Vol.280, no.3: 222-224. Available at: https://doi.org/10.1001/jama.280.3.222.

Franceschet, M. and Costantini, A. 2010. The effect of scholar collaboration on impact and quality of academic papers. Journal of Informetrics, Vol.4, no.4: 540-553. Available at: https://doi.org/10.1016/j.joi.2010.06.003.

Garg, K. and Padhi, P. 2002. Scientometrics of laser research in India during 1970-1994. Scientometrics, Vol.55, no.2: 215-241. Available at: https://doi.org/10.1023/A:1019615723942.

Gazni, A., Sugimoto, C. and Didegah, F. 2012. Mapping world scientific collaboration: Authors, institutions, and countries. Journal of the American Society for Information Science and Technology, Vol.63, no.2: 323-335.

Green, B.N. and Johnson, C.D. 2015. Interprofessional collaboration in research, education, and clinical practice: working together for a better future. The Journal of Chiropractic Education, Vol.29, no.1: 1-10. Available at: https://doi.org/10.7899/JCE-14-36. 
Greene, M. 2007. The demise of the lone author. Nature, Vol.450, no.7173: 1165. Available at: https://doi.org/10.1038/4501165a.

Hall, K.L., Feng, A.X., Moser, R.P., Stokols, D. and Taylor, B.K. 2008. Moving the science of team science forward: Collaboration and creativity. American Journal of Preventive Medicine, Vol.35, no.2: S243-S249.

Halperin, E.C., Scott, J. and George, S. 1992. Multiple authorship in two English-language journals in radiation oncology. Academic Medicine, Vol.67, no.12: 850-856. Available at: https://doi.org/10.1097/00001888-199212000-00012.

Hinnant, C.C., Stvilia, B., Wu, S., Worrall, A., Burnett, G., Burnett, K., Kazmer, M.M. and Marty, P.F. 2012. Author-team diversity and the impact of scientific publications: Evidence from physics research at a national science lab. Library and Information Science Research, Vol.34, no.4: 249-257. Available at: https://doi.org/10.1016/j.lisr.2012.03.001.

Hoen, W.P., Walvoort, H C. and Overbeke, A.J. 1998. What are the factors determining authorship and the order of the authors' names? A study among authors of the Nederlands Tijdschrift voor Geneeskunde (Dutch Journal of Medicine). JAMA, Vol.280, no.3: 217-218. Available at: https://doi.org/10.1001/jama.280.3.217.

Hotz, R.L. 2015. How many scientists does it take to write a paper? Apparently, thousands. Available at: https://www.wsj.com/articles/how-many-scientists-does-it-take-to-writea-paper-apparently-thousands-1439169200.

ICMJE. 2016. Defining the role of authors and contributors. Available at: http://www.icmje.org/recommendations/browse/roles-and-responsibilities/definingthe-role-of-authors-and-contributors.html.

Iribarren-Maestro, I., Lascurain-Sánchez, M. and Sanz-Casado, E. 2009. Are multi-authorship and visibility related? Study of ten research areas at Carlos III University of Madrid. Scientometrics, Vol.79, no.1: 191-200. Available at: https://doi.org/10.1007/s11192009-0412-4.

Jawaid, M. and Jawaid, S.A. 2013. Faculty member's views, attitude and current practice as regards International Committee of Medical Journal Editors Criteria for authorship. Iranian Journal of Public Health, Vol.42, no.10: 1092-1098.

Jia, Z., Wu, Y., Tang, Y., Ji, W., Li, W., Zhao, X., Li, H., He, Q. and Ruan, D. 2016. Equal contributions and credit: an emerging trend in the characterization of authorship in major spine journals during a 10-year period. European Spine Journal, Vol.25, no.3: 913917. Available at: https://doi.org/10.1007/s00586-015-4314-2.

Kapoor, N., Abola, M.V., Jena, A.B. and Smith, S.E. 2015. Trends in authorship patterns in highimpact radiology publications, 1980-2013. Academic Radiology, Vol.22, no.12: 15871591. Available at: https://doi.org/10.1016/j.acra.2015.08.013.

Kennedy, M.S., Barnsteiner, J. and Daly, J. 2014. Honorary and ghost authorship in nursing publications. Journal of Nursing Scholarship, Vol.46, no.6: 416-422. Available at: 
https://doi.org/10.1111/jnu.12093.

King, C. 2012. Multiauthor Papers: Onward and Upward. Available at: http://archive.sciencewatch.com/newsletter/2012/201207/multiauthor_papers/.

Kornhaber, R.A., Mclean, L.M. and Baber, R.J. 2015. Ongoing ethical issues concerning authorship in biomedical journals: An integrative review. International Journal of Nanomedicine, Vol.10: 4837-4846. Available at: https://doi.org/10.2147/IJN.S87585.

Kretschmer, H. and Rousseau, R. 2001. Author inflation leads to a breakdown of Lotka's Law. Journal of the American Society for Information Science and Technology, Vol.52, no.8: 610-614.

Laudel, G. 2002. What do we measure by co-authorships? Research Evaluation, Vol.11, no.1: 3-15. Available at: https://doi.org/10.3152/147154402781776961.

Leimu, R. and Koricheva, J. 2005. Does scientific collaboration increase the impact of ecological crticles? BioScience, Vol.55, no.5: 438-443.

Ma, N. and Guan, J. 2005. An exploratory study on collaboration profiles of Chinese publications in Molecular Biology. Scientometrics, Vol.65, no.3: 343-355.

Milojević, S. 2014. Principles of scientific research team formation and evolution. Proceedings of the National Academy of Sciences of the United States of America, Vol.111, no.11: 3984-3989.

Mitchell, M.B. 1961. Trends toward multiple authorship in scientific publications. The Journal of Psychology, Vol.52, no.1: 125-131.

Morris, S. and Goldstein, M. 2007. Manifestation of research teams in journal literature: A growth model of papers, authors, collaboration, coauthorship, weak ties, and Lotka's law. Journal of the American Society for Information Science and Technology, Vol.58, no.12: 1764-1782.

Mowatt, G., Shirran, L., Grimshaw, J.M., Rennie, D., Flanagin, A., Yank, V., MacLennan, G., Gøtzsche, P.C. and Bero, L.A. 2002. Prevalence of honorary and ghost authorship in Cochrane reviews. JAMA, Vol.287, no.21: 2769-2771.

Ojerholm, E. and Swisher-Mcclure, S. 2015. Authorship in radiation oncology: Proliferation trends over 30 years. International Journal of Radiation Oncology Biology Physics, Vol.93, no.4: 754-756.

Onodera, N. and Yoshikane, F. 2015. Factors affecting citation rates of research articles. Journal of the Association for Information Science and Technology, Vol.66, no.4: 739-764.

Osborne, J.W. and Holland, A. 2009. What is authorship, and what should it be? A survey of prominent guidelines for determining authorship in scientific publications. Practical Assessment, Research \& Evaluation, Vol.14, no.15: 1-19.

Papatheodorou, S.I., Trikalinos, T.A. and loannidis, J.P.A. 2008. Inflated numbers of authors over time have not been just due to increasing research complexity. Journal of Clinical Epidemiology, Vol.61, no.6: 546-551. 
Patience, G. S., Patience, C. A., Blais, B. and Bertrand, F. 2017. Citation analysis of scientific categories. Heliyon, Vol.3, no.5. Available at: https://doi.org/10.1016/j.heliyon.2017.e00300.

Pennock, R. T. 1996. Inappropriate authorship in collaborative science research. Public Affairs Quarterly, Vol.10, no.4: 379-393.

Price, D.J. de S. 1986. Little science, big science-- and beyond. New York: Columbia University Press.

Pritychenko, B. 2016. Fractional authorship in nuclear physics. Scientometrics, Vol.106, no.1: 461-468

Rajasekaran, S., Li Pi Shan, R. and Finnoff, J.T. 2014. Honorary authorship: Frequency and associated factors in physical medicine and rehabilitation research articles. Archives of Physical Medicine and Rehabilitation, Vol.95, no.3: 418-428.

Regalado, A. 1995. Multiauthor papers on the rise. Science, Vol.268, no.5207: 25.

Resnik, D.B. and Master, Z. 2011. Authorship policies of bioethics journals. Journal of Medical Ethics, Vol.37, no.7: 424-428. Available at: https://doi.org/10.1136/jme.2010.040675.

Resnik, D.B., Tyle, A.M., Black, J.R. and Kissling, G. 2016. Authorship policies of scientific journals. Journal of Medical Ethics, Vol.42, no.3: 199-202. Available at: https://doi.org/10.1136/medethics-2015-103171.

Rousseau, R. 1992. Breakdown of the robustness property of Lotka's law: The case of adjusted counts for multiauthorship attribution. Journal of the American Society for Information Science, Vol.43, no.10: 645-647.

Salager-Meyer, F., Ariza, M. and Berbesí, M. 2009. "Backstage solidarity" in Spanish- and English-written medical research papers: Publication context and the acknowledgment paratext. Journal of the American Society for Information Science and Technology, Vol.60, no.2: 307-317.

Slone, R.M. 1996. Coauthors' contributions to major papers published in the AJR: Frequency of undeserved coauthorship. American Journal of Roentgenology, Vol.167, no.3: 571579.

Tarnow, E., De Young, B.R. and Cohen, M.B. 2004. Coauthorship in pathology, a comparison with physics and a survery-generated and member-preferred authorship guideline. MedGenMed, Vol.6, no.3: 1-2. Available at: https://www.ncbi.nlm.nih.gov/pmc/articles/PMC1435638/.

Vera-Badillo, F.E., Napoleone, M., Krzyzanowska, M.K., Alibhai, S.M.H., Chan, A.W., Ocana, A., Templeton, A. J., Seruga, B., Amir, E. and Tannock, I.F. 2016. Honorary and ghost authorship in reports of randomised clinical trials in oncology. European Journal of Cancer, Vol.66: 1-8.

Vinther, S. and Rosenberg, J. 2012. Appearance of ghost and gift authors in Ugeskrift for Laeger and Danish Medical Journal. Danish Medical Journal, Vol.59, no.5: 1-6. 
Wager, E. 2007. Do medical journals provide clear and consistent guidelines on authorship? Medscape General Medicine, Vol. 9, no.3: 16.

Wislar, J.S., Flanagin, A., Fontanarosa, P.B. and Deangelis, C.D. 2011. Honorary and ghost authorship in high impact biomedical journals: a cross sectional survey. BMJ: British medical journal, Vol.343, no.7835. Available at: https://doi.org/10.1136/bmj.d6128.

Wuchty, S., Jones, B.F. and Uzzi, B. 2007. The increasing dominance of teams in production of knowledge. Science, Vol.316, no.5827: 1036-1039. Available at: https://doi.org/10.1126/science.1136099.

Yukawa, Y., Kitanaka, C. and Yokoyama, M. 2014. Authorship practices in multi-authored papers in the natural sciences at Japanese universities: Authorship practice in Japanese natural sciences. International Journal of Japanese Sociology, Vol.23, no.1: 80-91. 